\title{
Treatment of Acute Achilles Tendon Rupture
}

\author{
Seung-Hwan Park, MD, Ho Seong Lee, MD, Ki Won Young, MD*, Sang Gyo Seo, MD \\ Department of Orthopedic Surgery, Asan Medical Center, University of Ulsan College of Medicine, Seoul, \\ ${ }^{*}$ Department of Orthopedic Surgery, Eulji Hospital, Seoul, Korea
}

\begin{abstract}
There is no clear consensus on the optimal treatment of acute Achilles tendon rupture. Recently, studies have demonstrated the critical role of functional rehabilitation in the treatment of ruptured Achilles tendons. Hence, conservative treatment is preferred by a growing number of surgeons seeking to treat the condition without the risk of complications from surgery. However, operative treatment is still considered as a more reliable treatment option for acute Achilles tendon rupture. In this review article, we provide an overview of recent treatment strategies for acute rupture of the Achilles tendon.
\end{abstract}

Keywords: Achilles tendon, Injuries, Surgery, Rehabilitation

The Achilles tendon is the strongest and largest tendon in the body, but it is also the most commonly ruptured tendon. The overall incidence of Achilles tendon rupture is on the rise recently ${ }^{1,2)}$ because of the aging of the population, growing prevalence of obesity, and increased participation in sports. ${ }^{3)}$

Controversy has surrounded the optimal treatment of acute Achilles tendon rupture. ${ }^{4)}$ In the past, aggressive surgical intervention was recommended over conservative management on the basis of early studies that associated conservative treatment with high rerupture rates. ${ }^{5-8)}$ These studies provided a rationale for operative treatment of acute rupture of the Achilles tendon, despite the risk of complications from surgery such as wound infection. However, recent studies have demonstrated favorable outcomes of conservative treatment using accelerated functional rehabilitation. In such studies, functional rehabilitation was more effective in reducing rerupture rates than long-term cast immobilization, and functional improvement after nonoperative treatment was comparable to that after operative repair. ${ }^{9-11)}$ Currently, regardless of the treatment modality-either conservative or opera-

Received August 20, 2019; Accepted October 31, 2019

Correspondence to: Sang Gyo Seo, MD

Department of Orthopedic Surgery, Asan Medical Center, University of Ulsan

College of Medicine, 88 Olympic-ro 43-gil, Songpa-gu, Seoul 05505, Korea

Tel: +82-2-3010-3530, Fax: +82-2-488-7877

E-mail: hosng@amc.seoul.kr tive- used, aggressive early rehabilitation is advocated for acute Achilles tendon ruptures to allow for an early return to activities of daily living, high patient satisfaction, and functional improvement. In this review article, we provide a comprehensive review of the literature on acute rupture of the Achilles tendon and discuss appropriate treatment options.

\section{EPIDEMIOLOGY}

Achilles tendon rupture accounts for $20 \%$ of all large tendon ruptures. ${ }^{12)}$ The estimated incidence ranges from 11 to 37 per 100,000 population. ${ }^{13-15)}$ Men are 2 to 12 times more prone to Achilles tendon rupture than women. ${ }^{16)}$ In a 2012 meta-analysis by Soroceanu et al., ${ }^{10)}$ the mean age at the time of injury among 826 patients with an acute Achilles tendon rupture was 39.8 years. The injury has a bimodal age distribution with the first peak in patients between 25 years and 40 years of age and the second peak in those over 60 years. ${ }^{17,18)}$ High-energy injuries in sports are responsible for the first peak, whereas the second peak occurring in the elderly is mostly associated with low-energy injuries, such as spontaneous rupture of the degenerated Achilles tendon or rupture in chronic Achilles tendinopathy. In young patients with acute sports injures, conservative management is usually sufficient for tendon healing. However, rupture of the degenerated tendon in the elderly requires a different treatment approach because the tendon remains vulnerable to rerupture even after operative 
repair. Therefore, it is important to differentiate acute rupture of the Achilles tendon from rupture of the degenerated tendon.

\section{ANATOMY}

The Achilles tendon is the largest and strongest tendon in the body. ${ }^{19)}$ Tendinous fibers of the gastrocnemius originating from the distal femur and those of the soleus muscle originating from the proximal tibia coalesce above the insertion on the posterior calcaneal tuberosity. ${ }^{20)}$ The approximately $15-\mathrm{cm}$-long Achilles tendon travels distally and twists approximately $90^{\circ}$ internally so that the initially anterior fibers of the gastrocnemius insert laterally and the initially posterior fibers of the soleus insert on the medial aspect of the Achilles tendon. The Kager's fat pad located anterior to the Achilles tendon protects blood vessels entering the tendon. ${ }^{21)}$

The Achilles tendon has no tendon sheath but a highly vascularized paratenon ${ }^{22)}$ that acts as a conduit for the vasculature of the tendon and facilitates tendon gliding between the subcutaneous tissue and posterior fascia. ${ }^{22)}$ The proximal and distal sections of the tendon are supplied by the posterior tibial artery and the midsection ( 2 to $6 \mathrm{~cm}$ from the insertion point) is supplied by the peroneal artery. ${ }^{23)}$ Since the midsection receives a relatively poor blood supply, it is most vulnerable to degeneration and rupture. $^{24)}$

\section{DIAGNOSIS}

\section{Clinical Findings}

The diagnosis of acute Achilles tendon rupture is mostly based on a thorough history taking and physical examination. Typical patients are in their third or fourth decade of life and present with sudden inability to walk and acute pain during running or jumping. Patients with an acute rupture of the tendon often describe that they heard a popping sound in the back of the leg in dorsiflexion of the ankle or had the feeling of being kicked in the back of the ankle. Signs of a ruptured tendon include plantar flexion weakness, difficulty with weight-bearing ambulation, and limping. A false-negative Thompson test result can occur if plantar flexion is produced by intact extrinsic foot flexors; approximately $25 \%$ of acute ruptures are initially neglected for this reason. ${ }^{25)}$

According to the American Academy of Orthopaedic Surgeons clinical practice guidelines, the diagnosis of an acute Achilles tendon rupture can be established by two or more of the following physical examination tests:
(1) a positive Thompson test, (2) decreased plantar flexion strength, (3) presence of a palpable defect, and (4) increased passive ankle dorsiflexion with gentle manipulation. ${ }^{26)}$ During diagnosis, it is important to differentiate traumatic sports injuries from low-energy injuries (Fig. 1). The latter is often associated with the degenerative process of the tendon, chronic tendinosis, a history of steroid injection, and older age.

\section{Radiological Findings}

The diagnosis of acute Achilles tendon rupture is primarily clinical, supported by imaging tests. Magnetic resonance imaging (MRI) or ultrasonography can be useful as a confirmatory test. Since MRI is not a dynamic imaging modality, it is not reliable in adequately determining partial or complete rupture. By contrast, ultrasonography is more effective in identifying the location of a tear, gap between the torn ends of the tendon, and partial/complete rupture. ${ }^{27}$ Plain radiography (lateral views of the ankle) is used in treatment planning. It also aids in identification of tendon swelling and increased soft tissue density in the Kager's fat pad. Above all, it is superior to other imaging modalities in detecting presence of a calcific lesion, Haglund prominence, or calcaneus avulsion fracture, suggestive of preexisting degeneration or chronic tendinosis (Fig. 2). In the case of a rupture in chronic Achilles tendinopathy, the risk of rerupture is high after either conservative treatment or direct repair. Direct healing of the pathologic tissue at the ruptured ends is oftentimes not feasible in this case. Then, the surgeon should consider other treatment options using healthy tissue, such as tendon reconstruction. Therefore, it is of utmost importance to confirm the presence or absence of pre-existing tendinopathy for differential diagno-

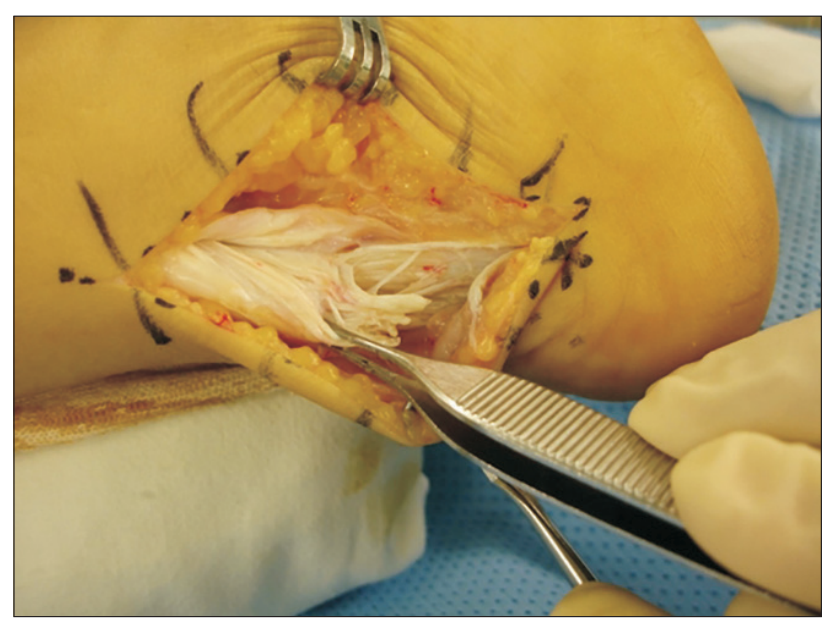

Fig. 1. Degenerated fibers of an Achilles tendon with chronic tendinitis. 


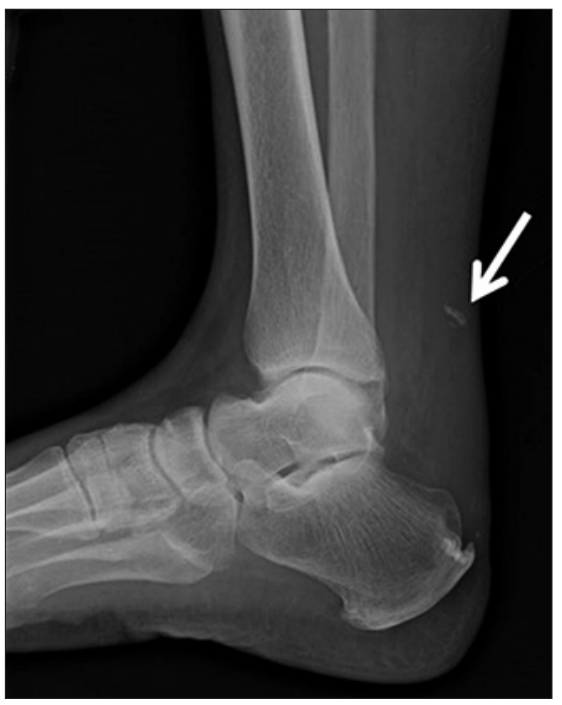

Fig. 2. Calcaneal spur and calcification (arrow) were observed in the degenerated Achilles tendon.

sis of acute Achilles tendon rupture. Plain radiography is a more appropriate than MRI or ultrasonography for this purpose.

\section{CONSERVATIVE TREATMENT}

There is still controversy over which treatment-conservative or operative-is more effective for acute Achilles tendon rupture. The concern related to conservative management is whether healing of a ruptured tendon is feasible without direct contact with surrounding structures. Delayed healing can result in calf weakness, and incomplete healing may increase the risk of rerupture. Bae et al. ${ }^{28)}$ reported that the tendon healed without direct contact of the ruptured ends despite the presence of a large defect. Although the study was based on the premise that the tissue at the margin of the ruptured ends should remain healthy, not pathologic, it supported the feasibility of tendon healing without direct repair.

The conventional conservative treatment entails 6 to 8 weeks of cast immobilization. The ankle is placed in a cast in plantar flexion position for initial 4 weeks and in neutral position for the following 2 to 4 weeks. Conservative management has been associated with a higher rerupture rate compared with operative repair (12.6\% vs. $3.5 \%){ }^{6}{ }^{6}$ However, recent studies have suggested that the rerupture rates can be lowered by reducing the period of cast immobilization and using early functional rehabilitation. ${ }^{11,29)}$ Rehabilitation is an integral part of treatment, either conservative or operative. Hence, much effort is focused on the development of optimal rehabilitations strategies.

\section{FUNCTIONAL REHABILITATION IN CONSERVATIVE TREATMENT}

In nonoperative treatment, rehabilitation is essential to treatment success. In the past, range of motion exercises and weight bearing after conservative treatment were not allowed as early as those after operative repair. However, recent studies underscore the benefits of early rehabilitation after conservative treatment of acute Achilles tendon rupture. $^{30)}$

As important as early rehabilitation after cast immobilization is the timely application of a functional walking brace. In a prospective randomized study, Saleh et al. ${ }^{31)}$ compared 8-week cast immobilization with 3-week cast immobilization followed by early mobilization in a functional brace. They found the use of a functional brace led to more rapid improvement of ankle dorsiflexion and earlier return to normal activities. Various rehabilitation protocols for conservative management of Achilles tendon ruptures are well documented in the literature. ${ }^{11,32-35)}$ Conservative management should not be misconstrued as "no treatment." Validated functional rehabilitation protocols are an integral part of conservative management of acute Achilles tendon rupture. These protocols should be effective when implemented in informed and cooperative patients; for uncooperative patients, operative repair should be considered as a treatment option. For the initial 8 weeks after an acute Achilles tendon injury, patients are required to wear a brace to prevent hyperdorsiflexion. Unfortunately, in Korea where people do not wear shoes indoors, patient's adherence to the use of a brace is relatively low; therefore, judicious patient selection is advised. Until 6 months after injury, low-impact activities are performed in a progressive manner; after 6 months, high-impact activities, such as soccer and basketball, are allowed. ${ }^{11)}$ To promote recovery to preinjury level of calf muscle strength, rehabilitation should be reasonably aggressive during the first year after injury, especially, for the first 6 months. It is because the recovery of calf muscle strength cannot be ensured with either conservative or operative treatment once 1 year has passed after injury. In a study by Lantto et al., ${ }^{36)}$ calf muscle strength in patients with acute Achilles tendon rupture did not recover to the normal level even at 11-years' follow-up and the isokinetic strength changed minimally between 1 year and 11 years of follow-up.

\section{OPERATIVE TREATMENT}

Various operative procedures for acute Achilles tendon rupture are described in the literature. They can be broadly categorized into open, mini-open, and percutaneous 
repair. ${ }^{37)}$ Among those, the posteromedial approach has been most frequently used since the hypervascularity on the medial side of the Achilles tendon was confirmed by angiography in a cadaver study. ${ }^{38)}$ The surgeon can determine repair techniques other than the Krackow suture method on the basis of his or her preference. Irrespective of the operative approach, however, one should restore the proper length of the tendon, guarding against excessive elongation. In general, the proper length is determined during surgery by comparing with the intact plantaris tendon; however, if the plantaris tendon is absent, the range of dorsiflexion on the contralateral side should be measured before draping or during surgery to use as a guide. After that, to prevent an infection, the paratenon surrounding the Achilles tendon should be repaired. While the open technique has shown good clinical results, it has also been associated with superficial and deep wound complications requiring reoperation. ${ }^{37}$

Percutaneous repair can be done by using multiple puncture wounds. A suture is woven through the proximal and distal portions of the tendon via puncture wounds. The suture is tied, bringing tendon ends into apposition in plantar flexion of the ankle. Blindly passing the suture in percutaneous repair can cause a sural nerve injury. The mini-open repair technique has been developed to minimize the complications such as postoperative wound infection of open repair and sural nerve injury in percutaneous repair. A small skin incision over the rupture site is made and subcutaneous soft-tissue is bluntly spread. Various devices are necessary for the mini-open repair technique. A device is introduced through the incision under the paratenon and the suture is passed from the external guide through the skin into the tendon and out to the opposite side. Usually, three sutures are passed through the proximal and distal tendon ends. The device and the suture are pulled out for apposition of ruptured tendon ends, and the sutures are tied with the ankle in plantar flexed position. Percutaneous repair with the mini-open technique, compared with open repair, results in lower wound complication rates and improves cosmetic appearance. However, overall complication rates are not significantly different between the mini-open, percutaneous repair and the open repair. ${ }^{37)}$

\section{FUNCTIONAL REHABILITATION IN OPERATIVE TREATMENT}

For tendon healing, early functional rehabilitation is more important than the surgery itself. Huang et al. ${ }^{39)}$ reported that early weight bearing combined with early ankle mo- tion exercises was more effective for postoperative recovery than the conventional immobilization or early ankle motion exercises alone. Brumann et al. ${ }^{40)}$ also emphasized the importance of accelerated rehabilitation. According to their rehabilitation protocol, full weight bearing in $30^{\circ}$ fixed plantar flexion is started immediately after surgery; controlled ankle mobilization in free plantar flexion and limited dorsiflexion at $0^{\circ}$, after the second postoperative week.

Prolonged postoperative immobilization is not desirable. In particular, more than 3 weeks of immobilization in a splint or cast should be avoided. Long-leg cast immobilization is no longer recommended. Full weight bearing in an orthosis is initiated immediately after surgery or at least within 3 weeks after surgery, and it should be worn for 6 to 8 postoperative weeks. Although the use of a removable brace is allowed for early range of motion exercises, the patient should be cautioned to avoid hyperdorsiflexion of the ankle.

Although there is a broad consensus on the importance of early weight bearing, postoperative ankle position still remains the subject of debate. In general, the ankle is initially maintained in plantar flexion position with gradual dorsiflexion. However, some authors recommend neutral ankle position immediately after surgery to allow for full weight bearing, ${ }^{41)}$ because rerupture occurs frequently in gradual dorsiflexion of the plantar-flexed ankle during rehabilitation. Ryu et al. ${ }^{41)}$ reported there was no case of rerupture in the total 112 patients who started weight-bearing ambulation in neutral ankle position immediately after surgery. Regardless of the postoperative ankle position, however, it is important to avoid tendon elongation. An elongated Achilles tendon characterized by hyperdorsiflexion of the ankle in physical examination (Fig. 3) has been associated with plantar flexor weakness and functional deficit.

\section{COMPLICATIONS}

Complications of operative treatment of acute Achilles tendon rupture include sural nerve injury, infection, rerupture, deep vein thrombosis, and hypertrophic scars. Therefore, operative treatment may not be appropriate for low-demand patients or those with diabetes mellitus or peripheral vascular disease.

\section{Infection}

The most serious complication of open repair is infection. Infection and wound problems mostly occur after surgery with an incidence of $12.5 \% .{ }^{10,42)}$ To prevent an infection, 
Park et al. Acute Achilles Tendon Rupture

Clinics in Orthopedic Surgery • Vol. 12, No. 1, $2020 \bullet$ www.ecios.org

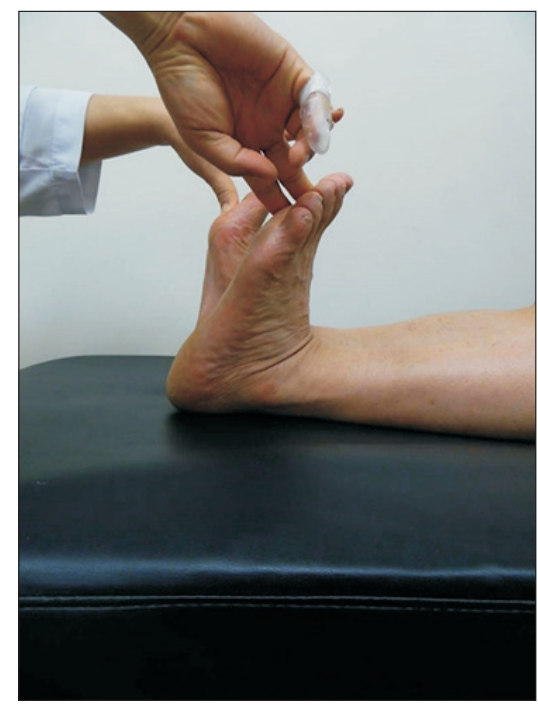

Fig. 3. Hyperdorsiflexed left ankle of a patient with the elongated Achilles tendon after an acute rupture.

the surgeon should avoid superficial dissection during incision and restore the synovial tissue envelope as much as possible before repair of the paratenon. In addition, one should use the minimal number of sutures to obviate delayed infection around the subcutaneous suture knot. Since the sutures need to hold the tendon for approximately 3 months of healing period after repair, absorbable sutures are preferable to nonabsorbable sutures, which increase the risk of delayed infection or irritation.

\section{Calf Muscle Weakness}

Patients are able to walk even without proper healing of a ruptured Achilles tendon; however, a permanent functional deficit will remain. Therefore, the ultimate goal of treatment is to prevent residual calf muscle weakness. The ability to perform a single heel raise is a valid indicator of calf muscle weakness; indeed, most patients with a neglected tear are unable to perform a single heel raise. ${ }^{43,44)}$

\section{Rerupture}

One of the most important considerations in selecting operative versus nonoperative treatment is the risk of rerupture. Rettig et al. ${ }^{45)}$ reported that the overall postoperative rerupture rate was $4.5 \%$ in their patients, and $16.6 \%$ of which occurred in those aged 30 years or younger. They suggested that caution should be observed during aggressive rehabilitation in younger patients. Reito et al. ${ }^{46)}$ also reported a rerupture rate of $7.1 \%$ in 210 patients with Acute Achilles tendon rupture after conservative treatment. The complication occurred within 12 weeks after treatment in most cases, and they suggested extra care should be taken in the first month after nonoperative treatment. Young et al. ${ }^{47)}$ noted that nine of the total 12 reruptures $(75 \%)$ occurred within 3 months after surgery and there was no association between the rerupture rate and the repair method.

\section{PROGNOSIS}

In general, patients will resume normal ambulation within 12.5 to 18 weeks after an acute rupture of the Achilles tendon, ${ }^{48)}$ but there is no doubt that early weight bearing and rehabilitation contribute to improved prognosis. ${ }^{11,29,48}$ Patients are conventionally advised against running and non-contact sports for 16 to 20 weeks after injury. ${ }^{49)}$ The criteria for return to running suggested by Van Sterkenburg et al ${ }^{50)}$ include the ability to perform repetitive single heel raises and toe walking and $\leq 25 \%$ calf strength deficit compared to the normal contralateral side, which should be met approximately 12 weeks after injury. Olsson et al. ${ }^{51)}$ also reported the heel raise ability as an important indicator of general level of healing. In their study, 40 out of 81 patients (49\%) with acute Achilles tendon ruptures were unable to perform a single heel raise at 12 weeks after the injury. In a study by Ryu et al., ${ }^{41)} 87$ of 112 patients with acute Achilles tendon ruptures had difficulty with a single heel raise at 3 months after open tenorrhaphy followed by early rehabilitation; however, all patients were able to raise the heel 6 months postoperatively.

McCormack and Bovard ${ }^{52)}$ noted a $10 \%$ to $30 \%$ calf strength deficit on the injured side compared to the uninjured side in their patients with acute Achilles tendon tears. Ryu et al. ${ }^{41)}$ also reported that even in patients who were able to perform single heel raises and sports after operative repair of acute tears and early rehabilitation, the calf circumference decreased by an average of $1.6 \mathrm{~cm}$ on the injured side and the isokinetic flexion peak torque deficit at $30 \%$ sec was $16 \%$ (range, $0 \%$ to $21 \%$ ) on the injured side compared with the uninjured side.

\section{CONCLUSIONS}

Acute Achilles tendon ruptures should be differentiated from ruptures that occur as the result of chronic degeneration of the tendon. An acute rupture of a healthy tendon can be successfully treated either conservatively or operatively. Irrespective of the treatment method, however, rehabilitation is a crucial component of treatment. Thus, patient's adherence to rehabilitation should be taken into consideration in determining a treatment strategy. Re- 
habilitation during the first 6 months after injury is of great importance for patients with an acute rupture of the Achilles tendon. While specifics of the rehabilitation protocol may vary, the focus of rehabilitation is on preventing rerupture for the first 2 months after injury and improving calf muscle strength for the next 1 month (between 2 months and 3 months after injury). Then, for the following 3 months (between 3 months and 6 months after injury), rehabilitation efforts are directed toward a return to sports through vigorous strengthening and proprioceptive exercises. Furthermore, during rehabilitation after either treatment, care should be taken not to cause hyperdorsiflexion of the ankle to prevent calf muscle weakness.

\section{CONFLICT OF INTEREST}

No potential conflict of interest relevant to this article was reported.

\section{REFERENCES}

1. Huttunen TT, Kannus P, Rolf C, Fellander-Tsai L, Mattila VM. Acute achilles tendon ruptures: incidence of injury and surgery in Sweden between 2001 and 2012. Am J Sports Med. 2014;42(10):2419-23.

2. Lantto I, Heikkinen J, Flinkkila T, Ohtonen P, Leppilahti J. Epidemiology of Achilles tendon ruptures: increasing incidence over a 33-year period. Scand J Med Sci Sports. 2015;25(1):e133-8.

3. Raikin SM, Garras DN, Krapchev PV. Achilles tendon injuries in a United States population. Foot Ankle Int. 2013;34(4):475-80.

4. Thevendran G, Sarraf KM, Patel NK, Sadri A, Rosenfeld P. The ruptured Achilles tendon: a current overview from biology of rupture to treatment. Musculoskelet Surg. 2013; 97(1):9-20.

5. Cetti R, Christensen SE, Ejsted R, Jensen NM, Jorgensen U. Operative versus nonoperative treatment of Achilles tendon rupture: a prospective randomized study and review of the literature. Am J Sports Med. 1993;21(6):791-9.

6. Khan RJ, Fick D, Keogh A, Crawford J, Brammar T, Parker M. Treatment of acute achilles tendon ruptures: a metaanalysis of randomized, controlled trials. J Bone Joint Surg Am. 2005;87(10):2202-10.

7. Moller M, Movin T, Granhed H, Lind K, Faxen E, Karlsson J. Acute rupture of tendon Achillis: a prospective randomised study of comparison between surgical and non-surgical treatment. J Bone Joint Surg Br. 2001;83(6):843-8.

8. Roberts CP, Palmer S, Vince A, Deliss LJ. Dynamised cast management of Achilles tendon ruptures. Injury. 2001;32(5):423-6.

9. Keating JF, Will EM. Operative versus non-operative treatment of acute rupture of tendo Achillis: a prospective randomised evaluation of functional outcome. J Bone Joint Surg Br. 2011;93(8):1071-8.

10. Soroceanu A, Sidhwa F, Aarabi S, Kaufman A, Glazebrook M. Surgical versus nonsurgical treatment of acute Achilles tendon rupture: a meta-analysis of randomized trials. J Bone Joint Surg Am. 2012;94(23):2136-43.

11. Willits K, Amendola A, Bryant D, et al. Operative versus nonoperative treatment of acute Achilles tendon ruptures: a multicenter randomized trial using accelerated functional rehabilitation. J Bone Joint Surg Am. 2010;92(17):2767-75.

12. Gillies $\mathrm{H}$, Chalmers J. The management of fresh ruptures of the tendo achillis. J Bone Joint Surg Am. 1970;52(2):337-43.

13. Houshian S, Tscherning T, Riegels-Nielsen P. The epidemiology of Achilles tendon rupture in a Danish county. Injury. 1998;29(9):651-4.

14. Jarvinen TA, Kannus P, Maffulli N, Khan KM. Achilles tendon disorders: etiology and epidemiology. Foot Ankle Clin. 2005;10(2):255-66.

15. Levi N. The incidence of Achilles tendon rupture in Copenhagen. Injury. 1997;28(4):311-3.

16. Movin T, Ryberg A, McBride DJ, Maffulli N. Acute rupture of the Achilles tendon. Foot Ankle Clin. 2005;10(2):331-56.

17. Moller A, Astron M, Westlin N. Increasing incidence of Achilles tendon rupture. Acta Orthop Scand. 1996;67(5): 479-81.

18. Nillius SA, Nilsson BE, Westlin NE. The incidence of Achilles tendon rupture. Acta Orthop Scand. 1976;47(1):118-21.

19. O'Brien M. The anatomy of the Achilles tendon. Foot Ankle Clin. 2005;10(2):225-38.

20. Rufai A, Ralphs JR, Benjamin M. Structure and histopathology of the insertional region of the human Achilles tendon. J Orthop Res. 1995;13(4):585-93.

21. Benjamin M, Moriggl B, Brenner E, Emery P, McGonagle D, Redman S. The "enthesis organ" concept: why enthesopathies may not present as focal insertional disorders. Arthritis Rheum. 2004;50(10):3306-13.

22. Lohrer H, Arentz S, Nauck T, Dorn-Lange NV, Konerding MA. The Achilles tendon insertion is crescent-shaped: 
Park et al. Acute Achilles Tendon Rupture

Clinics in Orthopedic Surgery • Vol. 12, No. 1, $2020 \bullet$ www.ecios.org

an in vitro anatomic investigation. Clin Orthop Relat Res. 2008;466(9):2230-7.

23. Chen TM, Rozen WM, Pan WR, Ashton MW, Richardson MD, Taylor GI. The arterial anatomy of the Achilles tendon: anatomical study and clinical implications. Clin Anat. 2009;22(3):377-85.

24. Gwynne-Jones DP, Sims M, Handcock D. Epidemiology and outcomes of acute Achilles tendon rupture with operative or nonoperative treatment using an identical functional bracing protocol. Foot Ankle Int. 2011;32(4):337-43.

25. Inglis AE, Sculco TP. Surgical repair of ruptures of the tendo Achillis. Clin Orthop Relat Res. 1981;(156):160-9.

26. Chiodo CP, Glazebrook M, Bluman EM, et al. Diagnosis and treatment of acute Achilles tendon rupture. J Am Acad Orthop Surg. 2010;18(8):503-10.

27. Maffulli N, Dymond NP, Regine R. Surgical repair of ruptured Achilles tendon in sportsmen and sedentary patients: a longitudinal ultrasound assessment. Int J Sports Med. 1990;11(1):78-84.

28. Bae SH, Lee HS, Seo SG, Kim SW, Gwak HC, Bae SY. Debridement and functional rehabilitation for Achilles tendon infection following tendon repair. J Bone Joint Surg Am. 2016;98(14):1161-7.

29. Nilsson-Helander K, Silbernagel KG, Thomee R, et al. Acute achilles tendon rupture: a randomized, controlled study comparing surgical and nonsurgical treatments using validated outcome measures. Am J Sports Med. 2010;38(11):2186-93.

30. Young SW, Patel A, Zhu M, et al. Weight-bearing in the nonoperative treatment of acute Achilles tendon ruptures: a randomized controlled trial. J Bone Joint Surg Am. 2014;96(13):1073-9.

31. Saleh M, Marshall PD, Senior R, MacFarlane A. The Sheffield splint for controlled early mobilisation after rupture of the calcaneal tendon: a prospective, randomised comparison with plaster treatment. J Bone Joint Surg Br. 1992;74(2):2069.

32. Hutchison AM, Topliss C, Beard D, Evans RM, Williams P. The treatment of a rupture of the Achilles tendon using a dedicated management programme. Bone Joint J. 2015;97(4):510-5.

33. Ecker TM, Bremer AK, Krause FG, Muller T, Weber M. Prospective use of a standardized nonoperative early weightbearing protocol for achilles tendon rupture: 17 years of experience. Am J Sports Med. 2016;44(4):1004-10.

34. Barfod KW, Bencke J, Lauridsen HB, Ban I, Ebskov L, Troelsen A. Nonoperative dynamic treatment of acute achilles tendon rupture: the influence of early weight-bearing on clinical outcome: a blinded, randomized controlled trial. J Bone Joint Surg Am. 2014;96(18):1497-503.

35. Kauwe M. Acute Achilles tendon rupture: clinical evaluation, conservative management, and early active rehabilitation. Clin Podiatr Med Surg. 2017;34(2):229-43.

36. Lantto I, Heikkinen J, Flinkkila T, et al. Early functional treatment versus cast immobilization in tension after achilles rupture repair: results of a prospective randomized trial with 10 or more years of follow-up. Am J Sports Med. 2015;43(9):2302-9.

37. Hsu AR, Jones CP, Cohen BE, Davis WH, Ellington JK, Anderson RB. Clinical outcomes and complications of percutaneous Achilles repair system versus open technique for acute Achilles tendon ruptures. Foot Ankle Int. 2015;36(11):1279-86.

38. Yepes H, Tang M, Geddes C, Glazebrook M, Morris SF, Stanish WD. Digital vascular mapping of the integument about the Achilles tendon. J Bone Joint Surg Am. 2010;92(5):1215-20.

39. Huang J, Wang C, Ma X, Wang X, Zhang C, Chen L. Rehabilitation regimen after surgical treatment of acute Achilles tendon ruptures: a systematic review with meta-analysis. Am J Sports Med. 2015;43(4):1008-16.

40. Brumann M, Baumbach SF, Mutschler W, Polzer H. Accelerated rehabilitation following Achilles tendon repair after acute rupture: development of an evidence-based treatment protocol. Injury. 2014;45(11):1782-90.

41. Ryu CH, Lee HS, Seo SG, Kim HY. Results of tenorrhaphy with early rehabilitation for acute tear of Achilles tendon. J Orthop Surg (Hong Kong). 2018;26(3):2309499018802483.

42. Kadakia AR, Dekker RG 2nd, Ho BS. Acute Achilles tendon ruptures: an update on treatment. J Am Acad Orthop Surg. 2017;25(1):23-31.

43. Elias I, Besser M, Nazarian LN, Raikin SM. Reconstruction for missed or neglected Achilles tendon rupture with $\mathrm{V}-\mathrm{Y}$ lengthening and flexor hallucis longus tendon transfer through one incision. Foot Ankle Int. 2007;28(12):1238-48.

44. Yasuda T, Shima H, Mori K, Kizawa M, Neo M. Direct repair of chronic Achilles tendon ruptures using scar tissue located between the tendon stumps. J Bone Joint Surg Am. 2016;98(14):1168-75.

45. Rettig AC, Liotta FJ, Klootwyk TE, Porter DA, Mieling P. Potential risk of rerupture in primary achilles tendon repair in athletes younger than 30 years of age. Am J Sports Med. 2005;33(1):119-23.

46. Reito A, Logren HL, Ahonen K, Nurmi H, Paloneva J. Risk factors for failed nonoperative treatment and rerupture in acute Achilles tendon rupture. Foot Ankle Int. 
2018;39(6):694-703.

47. Young KW, Lee HS, Park SC. Rerupture risk after tenorrhaphy for Achilles tendon rupture. In: Proceedings of the 32nd Annual Meeting of Korean Orthopedic Society for Sports Medicine; 2018 Sep 15; Seoul, Korea. Daejeon: Korean Orthopaedic Society for Sports Medicine; 2018.

48. Costa ML, Donell ST, Tucker K. The long-term outcome of tendon lengthening for chronic Achilles tendon pain. Foot Ankle Int. 2006;27(9):672-6.

49. Maffulli N, Longo UG, Maffulli GD, Khanna A, Denaro V. Achilles tendon ruptures in elite athletes. Foot Ankle Int. 2011;32(1):9-15.
50. van Sterkenburg MN, Kerkhoffs GM, van Dijk CN. Good outcome after stripping the plantaris tendon in patients with chronic mid-portion Achilles tendinopathy. Knee Surg Sports Traumatol Arthrosc. 2011;19(8):1362-6.

51. Olsson N, Karlsson J, Eriksson BI, Brorsson A, Lundberg M, Silbernagel KG. Ability to perform a single heel-rise is significantly related to patient-reported outcome after Achilles tendon rupture. Scand J Med Sci Sports. 2014;24(1):152-8.

52. McCormack R, Bovard J. Early functional rehabilitation or cast immobilisation for the postoperative management of acute Achilles tendon rupture? A systematic review and meta-analysis of randomised controlled trials. Br J Sports Med. 2015;49(20):1329-35. 